\title{
Infrared-absorption coefficient for shallow donors in a quantum well
}

\author{
P. D. Emmel \\ Departamento de Física, Universidade Federal de São Carlos, via Washington Luiz km 235, \\ 13560 São Carlos, São Paulo, Brazil \\ and Instituto de Física, Universidade de São Paulo, Caixa Postal 20516, 01498 \\ São Paulo, São Paulo, Brazil \\ J. R. Leite \\ Instituto de Física, Universidade de São Paulo, Caixa Postal 20516, 01498 \\ São Paulo, São Paulo, Brazil \\ I. C. da Cunha Lima \\ Instituto de Física, Universidade de São Paulo, Caixa Postal 20516, 01498 \\ São Paulo, São Paulo, Brazil \\ and Instituto de Pesquisas Espaciais, Caixa Postal 515, 12201 Săo José dos Campos, \\ São Paulo, Brazil \\ (Received 5 July 1990; revised manuscript received 26 November 1990)
}

\begin{abstract}
We present a calculation of the absorption coefficient for the intraimpurity transitions between the first hydrogenlike donor states $(n \leq 3)$ existing in a quantum well (QW) of finite width, for uniform, Gaussian-, and $\delta$-like dopings. The eigenstates are obtained using a variational formulation with Slater-type functions. In this way we obtain the states $1 s, 2 s, 3 s, 3 p_{ \pm}$, and $3 d 2_{ \pm}$. The obtained wave functions allow us to calculate the oscillator strengths of the transitions, resulting in the absorption coefficient, which is in good agreement with the experimental values available in the literature.
\end{abstract}

$\mathrm{Ga}_{1-x} \mathrm{Al}_{x} \mathrm{As} / \mathrm{GaAs}$ quantum wells (QW's) are presently a popular system to study transport and optical properties of a quasi-two-dimensional (quasi-2D) electron gas. They are generated by several techniques by growing a layer of GaAs (narrow-gap region) between two layers of $\mathrm{Ga}_{1-x} \mathrm{Al}_{x} \mathrm{As}$ (wide-gap regions). For the undoped case, defined here as the absence of impurities in the GaAs, the electron in that region is confined by a potential barrier at the interfaces but is, nevertheless, free to move in the plane normal to the growing direction.

During the growth process donors and acceptors can be made to occur either inside or outside the GaAs layer in a very well controlled way. For a single donor impurity inside the well, an additional bound electron state appears below the first subband of the quasi-2D electron gas. That state is a hydrogenlike one, as in bulk semiconductors, perturbed by the confining potential in the $z$ direction. This break in symmetry makes the binding energy depend on the $z$ position of the impurity. In the low impurity concentration limit, the so-called dilute case, those bound states are important in determining the infrared properties of the heterostructures. These states have been extensively studied experimentally by Shanabrook and co-workers. ${ }^{1,2}$

On the other hand, the calculation of the energy levels of an electron bound to an impurity in a quantum well has been performed by many authors. To our knowledge, the first one was due to Bastard, ${ }^{3}$ who used as the wave function of the ground state a Slater-type $1 s$ hydrogenlike function times the wave function of the ground state of the $\mathrm{QW}$. Using a calculation based on a single variation- al parameter he obtained the energy of an electron as a function of the position of the impurity. Bastard et al. ${ }^{4}$ also calculated the energy level of the $2 p_{x, y}$ states and the imaginary part of the dielectric function for the transition $1 s \rightarrow 2 p_{x, y}$. Later, Greene and Bajaj calculated the impurity energy levels in the presence of a magnetic field, using a basis of Gaussian-type orbitals. ${ }^{5-7}$ In a review article, Shanabrook ${ }^{8}$ lists an extensive bibliography on hydrogenlike impurity levels. In this work we add to these results the calculation of the states $2 s, 3 s, 3 p_{ \pm}$, and $3 d 2_{+}$, using a set of Slater-type wave functions.

The system of an electron bound to an impurity inside an infinite $\mathrm{QW}$ is described by the following Hamiltonian:

$$
H=-\frac{\hbar^{2}}{2 m^{*}} \nabla^{2}-\frac{e^{2}}{K u}+V(z)
$$

where $u=\left[x^{2}+y^{2}+\left(z-z_{i}\right)^{2}\right]^{1 / 2}$ is the distance between the electron and the impurity located at $\left(0,0, z_{i}\right), m^{*}$ is the effective mass of the electron, $K$ is the effective dielectric constant, and $V(z)$ is the confining potential:

$$
V(z)=\left\{\begin{array}{cc}
0 & \text { if }|z|<L / 2 \\
\infty & \text { if }|z| \geq L / 2 .
\end{array}\right.
$$

Here $L$ is the well width. We assume the donor impurity located at $\left|z_{i}\right| \leq L / 2$.

In the case of a structure formed by GaAs and $\mathrm{Ga}_{1-x} \mathrm{Al}_{x} \mathrm{As}$, we neglect the image potential in view of the small difference between the two dielectric constants of the materials that form the QW. The wave functions are built as products of the ground-state wave function of 
the QW times hydrogenlike wave functions. Defining

$$
P_{n}(v)=1+a_{1}(L) v+a_{2}(L) v^{2}+\cdots+a_{n}(L) v^{n}
$$

we have, in cylindrical coordinates $(z, \rho, \varphi)$,

$$
\begin{aligned}
& \phi_{n s}(\kappa, \mathbf{r})=\Phi_{\mathrm{SB}}(z) P_{n-1}(\kappa u) e^{-\kappa u}, \\
& \phi_{n p_{ \pm}}(\kappa, \mathbf{r})=\Phi_{\mathrm{SB}}(z) \rho e^{ \pm i \varphi} P_{n-2}(\kappa u) e^{-\kappa u}, \\
& \phi_{n d 2_{ \pm}}(\kappa, \mathbf{r})=\Phi_{\mathrm{SB}}(z) \rho^{2} e^{ \pm 2 i \varphi} P_{n-3}(\kappa u) e^{-\kappa u},
\end{aligned}
$$

where

$$
\Phi_{\mathrm{SB}}(z)=\cos \left(\frac{\pi z}{L}\right)
$$

is the ground-state wave function of the QW.

In the above equations we used a single variational parameter, $\kappa$. In this paper we do not calculate states as $n p_{0}$ because, as shown by Greene and Bajaj, ${ }^{7}$ they become unbound as the QW width becomes sufficiently small. Therefore, we are concerned with states that stay bound in the two-dimension (2D) limit. These states are labeled by Ghazali, Gold, and Serre ${ }^{9}$ until $n=3$.

The coefficients $a_{j}(L)$ are determined in such a way that we have the correct 2D and 3D limits when $L$ tends to 0 and $\infty$, respectively. For example, the coefficient $a_{1}(L)$ that appears in $P_{1}(\kappa u)$ for the wave function $\phi_{2 s}(\kappa, \mathbf{r})$ has the limits -2 and -1 as $L$ goes to 0 and $\infty$, respectively, as we see from the hydrogen wave functions in 2D and 3D. We have chosen to express these coefficients as

$a_{j}(L)=\left(\frac{a_{j}^{3 \mathrm{D}}-a_{j}^{2 \mathrm{D}}}{2}\right) \tanh (L-5)+\left(\frac{a_{j}^{3 \mathrm{D}}+a_{j}^{2 \mathrm{D}}}{2}\right)$

in order to have a good fitting of the limits

$$
\begin{aligned}
& a_{j}^{3 \mathrm{D}} \equiv a_{j}(\infty), \\
& a_{j}^{2 \mathrm{D}} \equiv a_{j}(0)
\end{aligned}
$$

when $L \gg 5 a^{*}$ or $L<<5 a^{*}$ in each case, where $a^{*}$ is the effective Bohr radius, which for GaAs is $98 \AA$. However, we must point out that the results for the energies are not very sensitive to the choice of the value of $L$ corresponding to the crossing over. But we know that for $L \geq 10 a^{*}$ the $1 s$ state is already very close to the 3D hydrogen ground state. In Table I we give the limits of the coefficients used in this work.

As we must have orthogonal wave functions, we use the Schmidt process shown below:

$$
\begin{aligned}
& \Psi_{1 s}(\mathbf{r})=A_{1 s} \phi_{1 s}(\alpha, \mathbf{r}), \\
& \Psi_{2 s}(\mathbf{r})=A_{2 s}\left[\phi_{2 s}(\kappa, \mathbf{r})-\chi \Psi_{1 s}(\mathbf{r})\right], \\
& \Psi_{2 p_{ \pm}}(\mathbf{r})=A_{2 p_{ \pm}} \phi_{2 p_{ \pm}}(\gamma, \mathbf{r}), \\
& \Psi_{3 s}(\mathbf{r})=A_{3 s}\left[\phi_{3 s}(\eta, \mathbf{r})-\lambda \Psi_{1 s}(\mathbf{r})-\zeta \Psi_{2 s}(\mathbf{r})\right],
\end{aligned}
$$

TABLE I. Coefficients $a_{j}(L)$ for the limits $L \rightarrow 0$ and $L \rightarrow \infty$.

\begin{tabular}{ccccc}
\hline \hline$L$ & $a_{1}^{2 s}$ & $a_{1}^{3 s}$ & $a_{2}^{3 s}$ & $a_{1}^{3 p_{ \pm}}$ \\
\hline$\infty$ & -1 & -2 & $\frac{2}{3}$ & $-\frac{1}{2}$ \\
0 & -2 & -4 & 2 & $-\frac{2}{3}$ \\
\hline \hline
\end{tabular}

$$
\begin{aligned}
& \Psi_{3 p_{ \pm}}(\mathbf{r})=A_{3 p_{ \pm}}\left[\phi_{3 p_{ \pm}}(\mu, \mathbf{r})-\epsilon \Psi_{2 p_{ \pm}}(\mathbf{r})\right], \\
& \Psi_{3 d 2_{ \pm}}(\mathbf{r})=A_{3 d 2_{ \pm}} \phi_{3 d 2_{ \pm}}(v, \mathbf{r}) .
\end{aligned}
$$

In Fig. 1 we show the energy curves of the levels $1 s, 2 s, 2 p_{ \pm}, 3 s, 3 p_{ \pm}$, and $3 d 2_{ \pm}$as a function of $z_{i}$ for $L$ equal to $1 a^{*}$. We notice that the energies of the states with the same quantum number $n$ are nearly degenerate. The dispersion in energy decreases as $n$ (principal quantum number) increases. The binding energies of these states, for impurities at the center of the QW, versus the well width are in between $2 \mathrm{D}$ and $3 \mathrm{D}$ limits, as expected (Fig. 2).

In the dilute regime the optical transition between a determined pair of states is of the intrasite type, i.e., the transition occurs between two levels belonging to the same impurity. In this paper we study the absorption coefficient for several transitions possible. We are interested, then, in calculating the real part of the optical conductivity $\sigma_{1}(\omega)$ for these transitions.

Let $\sigma_{1}\left(z_{i}, \omega\right)$ be the contributions to $\sigma_{1}(\omega)$ due to an impurity located at $z_{i}$, for the transition between the levels $m$ and $n$ by absorption of a photon of frequency $\omega$ and whose wave vector is perpendicular to the QW's wall. We may write for the transition probability $W\left(z_{i}, \omega\right)$ per unity time $^{10}$

$$
\hbar \omega W\left(z_{i}, \omega\right)=\sigma_{1}\left(z_{i}, \omega\right)\left\langle\mathbf{E}^{2}\right\rangle .
$$

$W\left(z_{i}, \omega\right)$ is given by Fermi's golden rule,

$W\left(z_{i}, \omega\right)=\frac{2 \pi}{\hbar}\left|\left\langle n\left|\frac{-e}{K m^{*} c} \mathbf{A} \cdot \mathbf{p}\right| m\right\rangle\right|^{2} \delta\left(\Delta E\left(z_{i}\right)-\hbar \omega\right)$,

where

$$
\Delta E\left(z_{i}\right)=E_{n}\left(z_{i}\right)-E_{m}\left(z_{i}\right) .
$$

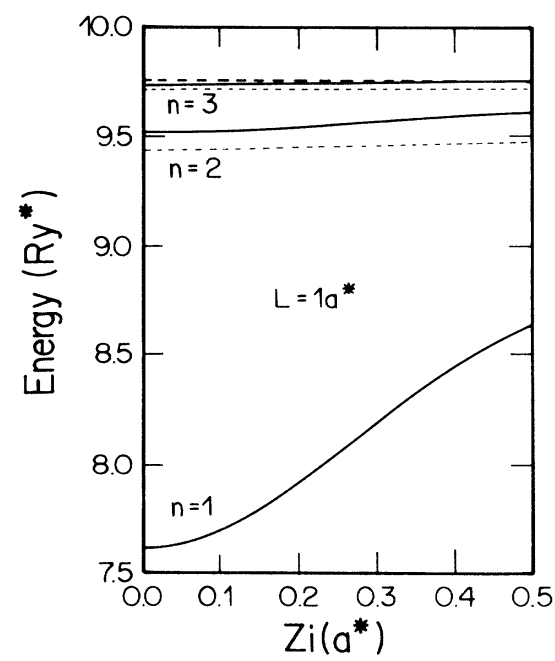

FIG. 1. Energy levels of states $1 s, 2 s, 2 p_{ \pm}, 3 s, 3 p_{ \pm}$, and $3 d 2_{ \pm}$for $L=1 a^{*}$. We characterize the $s$ states with solid lines, $p$ states with short-dashed lines, and the $3 d 2_{ \pm}$state with longdashed lines. 

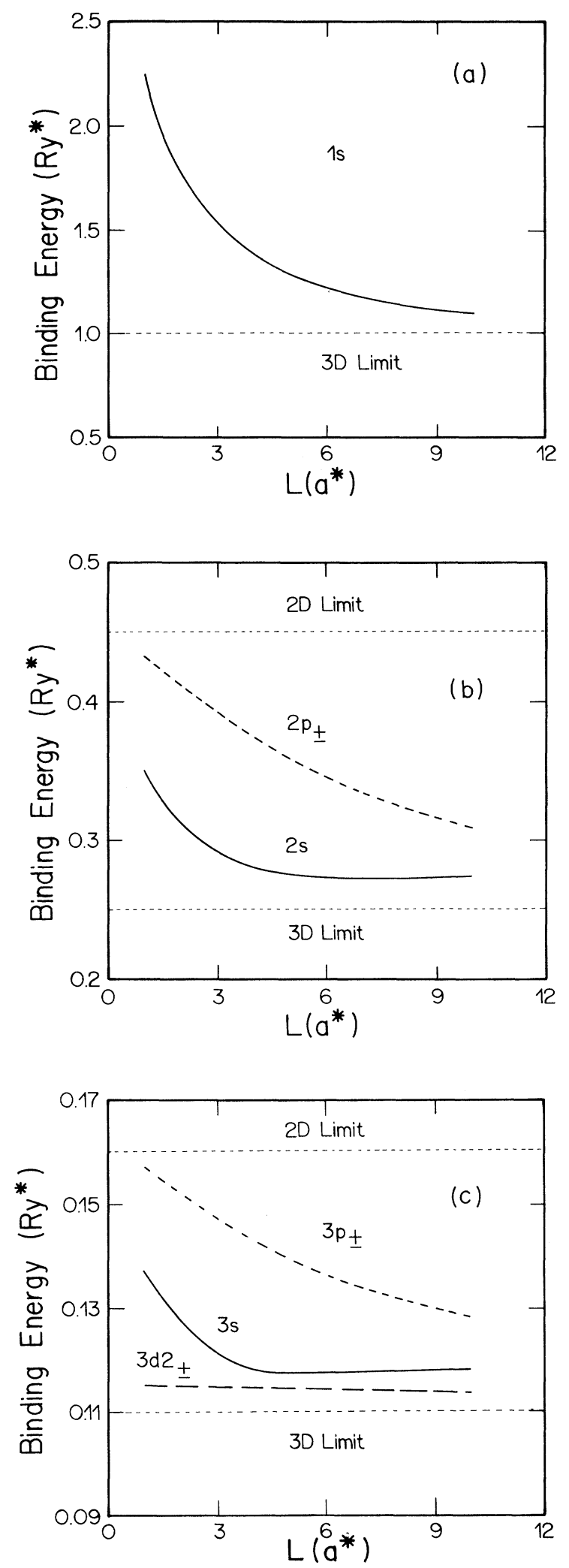

FIG. 2. Binding energies of an electron bound to an impurity at the center of the QW. (a) $1 s$ state, (b) $2 s$ and $2 p_{ \pm}$states, and (c) $3 s, 3 p_{ \pm}$, and $3 d 2_{ \pm}$states. The conventions are the same as above.

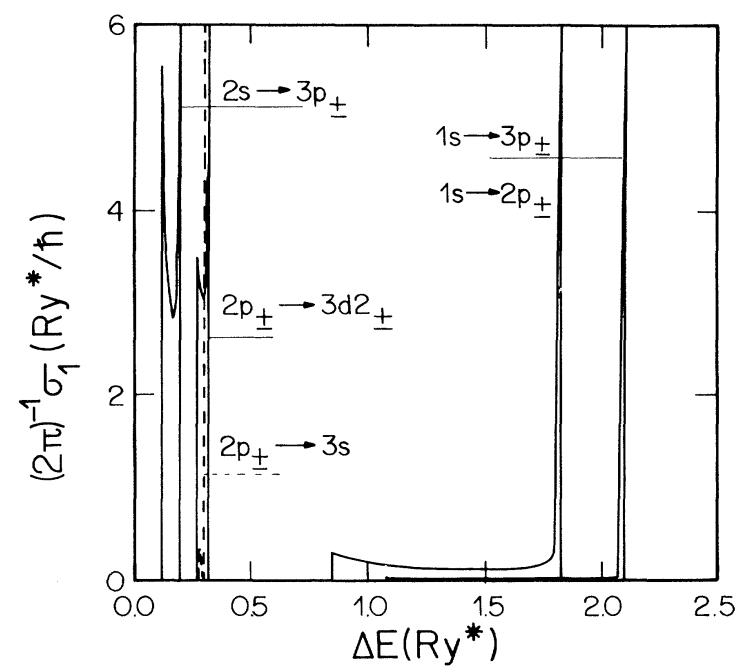

FIG. 3. Absorption coefficient for the $1 s \rightarrow 2 p_{ \pm}$, $1 s \rightarrow 3 p_{ \pm}, 2 p_{ \pm} \rightarrow 3 s, 2 p_{ \pm} \rightarrow 3 d 2_{ \pm}$, and $2 s \rightarrow 3 p_{ \pm}$transitions with $L=1 a^{*}$. The $2 p_{ \pm} \rightarrow 3 s$ transition is represented by a dashed line.

As

$$
\mathbf{A}(\mathbf{r}, t)=A_{0} \mathbf{u} e^{i(\mathbf{k} \cdot \mathbf{r}-\omega t)},
$$

where $\mathbf{u}$ is the polarization unit vector for the wave whose potential vector is $\mathbf{A}(\mathbf{r}, t)$ and

$$
\left\langle\mathbf{E}^{2}\right\rangle=\frac{E_{0}^{2}}{2}=\frac{1}{2} \frac{\omega^{2}}{c^{2}} A_{0}^{2},
$$

after performing a long-wavelength approximation, we have

$\sigma_{1}\left(z_{i}, \omega\right)=\pi \frac{e^{2}}{K} \omega|\mathbf{u} \cdot\langle n|\mathbf{r}| m\rangle|^{2} \delta\left(\Delta E\left(z_{i}\right)-\hbar \omega\right)$.

To obtain $\sigma_{1}(\omega)$, we must sum up the contributions of all impurities located in the QW. For the distribution of impurities $P\left(z_{i}\right)$ we have

$$
\begin{array}{rl}
\sigma_{1}(\omega)=\pi \frac{e^{2}}{K} \omega \int_{-L / 2}^{L / 2} & P\left(z_{i}\right)|\mathbf{u} \cdot\langle n|\mathbf{r}| m\rangle|^{2} \\
& \times \delta\left(\Delta E\left(z_{i}\right)-\hbar \omega\right) d z_{i} .
\end{array}
$$

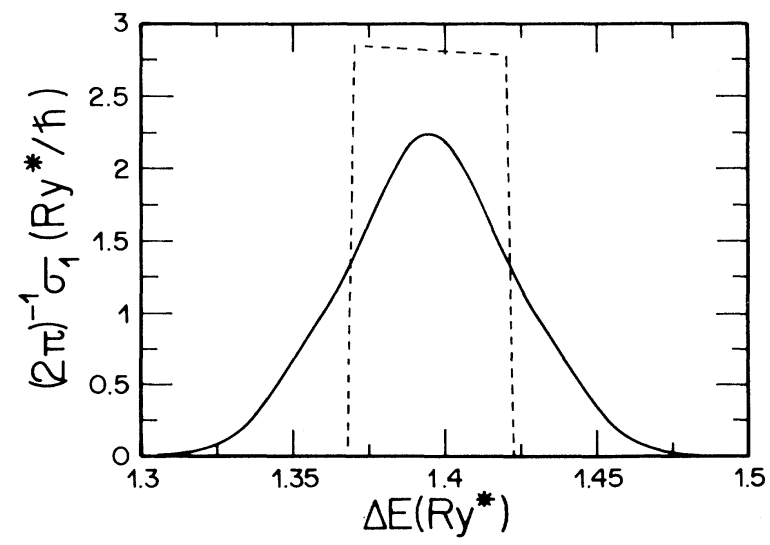

FIG. 4. Absorption coefficient for the $1 s \rightarrow 2 p_{ \pm}$transition with Gaussian (solid line) and $\delta$-like (dashed line) distributions for the doping width $d=0.02 a^{*}$ and $z_{m}=0.25 a^{*}$. 
But

$$
\delta\left(\Delta E\left(z_{i}\right)-\hbar \omega\right)=\sum_{l} \frac{\delta\left(z_{i}-z_{l}\right)}{\left|\frac{d \Delta E\left(z_{i}\right)}{d z_{i}}\right|_{\Delta E\left(z_{l}\right)=\hbar \omega}},
$$

where $z_{l}$ is the value of the continuous variable $z$ such that $\Delta E(z)=\hbar \omega$. Then

$$
\sigma_{1}(\omega)=\pi \frac{e^{2}}{K} \omega \sum_{l} \frac{P\left(z_{l}\right)|\mathbf{u} \cdot\langle n|\mathbf{r}| m\rangle|^{2}}{\left|\frac{d \Delta E\left(z_{i}\right)}{d z_{i}}\right|_{\Delta E\left(z_{l}\right)=\hbar \omega}} .
$$

\section{Calling}

$$
|\mathbf{u} \cdot\langle n|\mathbf{r}| m\rangle|^{2}=T_{n m}\left(\Delta E\left(z_{i}\right)\right)
$$

and remembering that $\Delta E\left(z_{i}\right)$ is an even function, we have

$$
\sigma_{1}(\omega)=\pi \frac{e^{2}}{K} \omega D(\hbar \omega) T_{n m}(\hbar \omega),
$$

where $D(\hbar \omega)$ is the joint density between the states $n$ and $m$. We see then that $\sigma_{1}(\omega)$ is obtained by weighing the $D(\hbar \omega)$ by the oscillator strength of the transition.

In this work we analyze three types of doping. First, the uniform one:

$$
P\left(z_{i}\right)=\frac{1}{L} .
$$

Figure 3 shows $\sigma_{1}(\omega)$ for the $1 s \rightarrow 2 p_{ \pm}, 1 s \rightarrow 3 p_{ \pm}$, $2 p_{ \pm} \rightarrow 3 s, 2 p_{ \pm} \rightarrow 3 d 2_{ \pm}$, and $2 s \rightarrow 3 p_{ \pm}$transitions with $L=1 a^{*}$.

As we can see, each transition presents two peaks. The peak corresponding to the highest energy is due to transitions occurring in the middle of the well. It is a consequence of the singularity of the joint density of states in that energy. The secondary peak comes from the impurities located at the edges of the well. The $1 s \rightarrow 2 p_{ \pm}$and $1 s \rightarrow 3 p_{ \pm}$transitions are broader than the others. Comparing our results with those experimental results obtained by Shanabrook and co-workers ${ }^{11}$ for the transition $1 s \rightarrow 2 p_{ \pm}$for the zero-magnetic-field limit, we obtain an error smaller than $4 \%$.

Other types of doping are the Gaussian- $\left[P_{G}\left(z_{i}\right)\right]$ and $\delta$-like $\left[P_{\delta}\left(z_{i}\right)\right]$ ones. We take distributions centered in $z_{m}$, with the width $d$, that is,

$$
\begin{aligned}
P_{G}\left(z_{i}\right) & =\frac{2}{\sqrt{2 \pi} d} \exp \left[-\frac{2\left(z_{i}-z_{m}\right)^{2}}{d^{2}}\right], \\
P_{\delta}\left(z_{i}\right) & = \begin{cases}1 / d & \text { if } z_{m}-d / 2<z_{i}<z_{m}+d / 2 \\
0 & \text { otherwise } .\end{cases}
\end{aligned}
$$

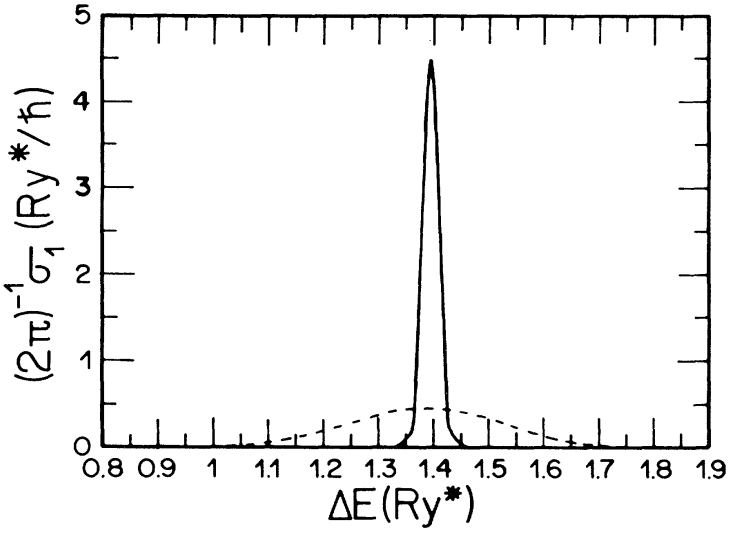

FIG. 5. Absorption coefficient for the $1 s \rightarrow 2 p_{+}$transition with Gaussian distribution for doping widths $d=0.1 a^{*}$ (dashed line) and $d=0.01 a^{*}$ (solid line), both for $z_{m}=0.25 a^{*}$.

The $\sigma_{1}(\omega)$ for the $\delta$-like distribution looks like a piece of $\sigma_{1}(\omega)$ for the uniform one, while for the Gaussian distribution we have a Gaussian structure. As we see in Fig. 4 these absorption coefficients do not present peaks, unless the doping is in the middle of the well. If the doping width becomes very narrow, there is an appearance of a peak, as is shown in Fig. 5. This fact suggests the manufacture of QW's with a sharp absorption spectra.

The energy levels have been obtained by a singleparameter variational calculation. The advantage of this strategy is a simplification on the integrals of energy and normalization constant as well as the computation of the extremes. In fact, the time spent in a two-parameter calculation increases without significant progress in calculation for the $1 s$ state. ${ }^{12}$ The price to be paid is the appearance of some strange features in one of our curves, as we can see in Fig. 3 for the absorption coefficients for the $2 p_{ \pm} \rightarrow 3 s$ transition, for $L=1 a^{*}$.

Summarizing, in this work we report on the results obtained for the absorption coefficients for three different types of impurity distributions on a QW. We have found that the absorption coefficients are strongly dependent on the analyzed impurity distributions. It is interesting to point out that a sharp peak is observed for this coefficient when the width of the distribution becomes thinner.

We would like to thank Dr. K. Watari for fruitful discussions on numerical methods for minimizing the computational time. One of us (P.D.E.) is grateful to Coordenação de Aperfeiçoamento de Pessoal do Ensino Superior for financial support.
${ }^{1}$ E. Glaser, B. V. Shanabrook, R. L. Hawkins, and W. Beard, Phys. Rev. B 36, 8185 (1987).

${ }^{2}$ E. Glaser, B. V. Shanabrook, R. J. Wagner, R. L. Hawkins, W. J. Moore, and D. Musser, J. Phys. (Paris) Colloq. 48, C5-239 (1987).

${ }^{3}$ G. Bastard, Phys. Rev. B 24, 4714 (1981).

${ }^{4}$ G. Bastard, E. E. Mendez, L. L. Chang, and L. Esaki, Solid State Commun. 45, 367 (1983).

${ }^{5}$ R. L. Greene and K. K. Bajaj, Solid State Commun. 45, 825 (1983).

${ }^{6}$ R. L. Greene and K. K. Bajaj, Phys. Rev. B 31, 913 (1985).
${ }^{7}$ R. L. Greene and K. K. Bajaj, Phys. Rev. B 37, 4604 (1988).

${ }^{8}$ B. V. Shanabrook, Physica B 146, 121 (1987).

${ }^{9}$ A. Ghazali, A. Gold, and J. Serre, Phys. Rev. B 39, 3400 (1989).

${ }^{10}$ J. N. Hodgson, Optical Absorption and Dispersion in Solids (Chapman and Hall, London, 1970).

${ }^{11}$ N. C. Jarosik, B. D. McCombe, B. V. Shanabrook, J. Comas, J. Ralston, and G. Wicks, Phys. Rev. Lett. 54, 1283 (1985).

${ }^{12}$ B. C. F. Colchesqui, P. D. Emmel, E. A. de Andrada e Silva, and I. C. da Cunha Lima, Phys. Rev. B 40, 12513 (1989). 